\title{
European Council of Vinyl Manufacturers Comments on Cicalese et al.: "An Ecological Study of the Association between Air Pollution and Hepatocellular Carcinoma Incidence in Texas"
}

\author{
Ariane Gennissen $^{a} \quad$ Marc Boeckx ${ }^{b, c}$ Arjen Sevenster ${ }^{c}$ \\ ${ }^{a}$ Arbo Unie, Rotterdam Europoort, Rotterdam, The Netherlands; ${ }^{b}$ VYNOVA, Tessenderlo,

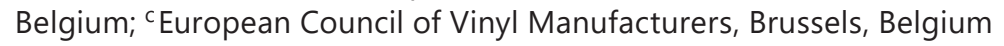

Dear Editor

The European Council of Vinyl Manufacturers (ECVM) wishes to comment on some aspects of the article by Cicalese et al. [1] in order to stress that the conclusions drawn by the authors are not properly substantiated. In particular, a link between increased risk of hepatocellular carcinoma (HCC) and vinyl chloride monomer (VCM) exposure is not demonstrated by the data cited in the study.

Before listing our main comments, we also would like to draw your attention to some minor but disturbing inaccuracies in the article:

- In the Abstract, hepatitis $\mathrm{C}$ and $\mathrm{B}$ are exchanged.

- In the section Data on page 289, the terms HCC incidence and liver cancer incidence are incorrectly exchanged. Indeed, it is liver cancer incidence which is reported by the Texas Cancer Registry, not HCC rates.

The authors used 4 sets of National Air Toxics Assessment (NATA) data for the years 1996, 1999, 2002, and 2005; these data were compared with HCC rates from 2000-2013 and 2006-2013. The median latency period for angiosarcoma of the liver and HCC is 36-48 years [2] rather than the duration of 7-13 years used in the study. The length of time between estimated VCM concentrations at county level and observation periods for HCC is, therefore, far too short. Comparing data from NATA 2005 with HCC observations in 20062013 entails especially a time frame of 1-8 years, rendering the association totally irrelevant.

The whole method regarding the data from the US Environmental Protection Agency (EPA) NATA, as presented on pages 290 and 291, is insufficiently described. As a consequence of this insufficient description, even though it is stated that "the analysis was conducted using data obtained from publicly available sources" [1], the reproducibility, replicability, and checking of the outcomes is hindered. The EPA NATA have actually described their methods extensively, and they are indeed publicly available, but what kind of data from the extensive NATA database are exactly used and how they are processed is unclear.

Marc Boeckx is Chair of the Medical Committee of the European Council of Vinyl Manufacturers. 
Last but not least, regarding the logistic regression method used, there is an essential bias in methodology. In the logistic regression method, a distinction/division is made between counties with a positive HCC rate and the other counties. The authors implicate that the distinguishing feature between the 2 groups is liver cancer. The methodologic confounder concerning this assumption is based on the fact that all zero and low rates (i.e., less than 16 cases) are suppressed. By doing this, the number of the population at risk has an essential distinguishing effect on whether a county is placed in the positive HCC rate group or the other group. The shorter the period, the more this confounder has an influence, thus having more effect on the 2006-2013 period than the 2000-2013 period. Hence, the conclusion that there is a limited association between any chemical evaluated and HCC incidence cannot be supported without further research into the effects of this bias on the results.

In conclusion, because of the methodological shortcomings and the unsubstantiated associations derived from statistical relationships, it is inappropriate to conclude that there is a significant positive association of HCC incidence with VCM exposure, and it is definitely inappropriate to state that there is an attributable cause-effect relationship. Furthermore, it is also definitely inappropriate and misleading to refer to such an alleged relationship in order to suggest that current VCM screening levels may not be protective.

\section{References}

1 Cicalese L, Raun L, Shirafkan A, et al: An ecological study of the association between air pollution and hepatocellular carcinoma incidence in Texas. Liver Cancer 2017;6:287-296.

2 Mundt KA, et al: Quantitative estimated exposure to vinyl chloride and risk of angiosarcoma of the liver and hepatocellular cancer in the US industry-wide vinyl chloride cohort: mortality update through 2013. Occup Environ Med 2017;74:709-716. 